\title{
First observation of quasi-2-day oscillations in ionospheric plasma frequency at fixed heights
}

\author{
D. Altadill ${ }^{1}$, J. G. Solé ${ }^{1}$, and E. M. Apostolov ${ }^{2}$ \\ ${ }^{1}$ Observatori de l'Ebre, CSIC, URL, Horta Alta 38, E-43520-Roquetes (Tarragona), Spain \\ ${ }^{2}$ Geophysical Institute, Bulgarian Academy of Sciences, Acad. G. Bonchev Str., bl. 3, 1113 Sofia, Bulgaria
}

Received: 23 July 1997 / Revised: 26 November 1997 / Accepted 2 December 1997

\begin{abstract}
The existence and development of the quasi2-day oscillations in the plasma frequency variations of the $\mathrm{F}$ region at northern middle latitudes are investigated. A new approach to study the quasi-2-day oscillations is presented, using a methodology that allows us to do such a study at fixed heights. The hourly values of plasma frequency at fixed heights, from $170 \mathrm{~km}$ to $220 \mathrm{~km}$ at $10 \mathrm{~km}$ step, obtained at the Observatori de l'Ebre station $\left(40.8^{\circ} \mathrm{N}, 0.5^{\circ} \mathrm{E}\right)$ during 1995 are used for analysis. It is found that quasi-2-day oscillations exist and persisted in the ionospheric plasma frequency variations over the entire year 1995 for all altitudes investigated. The dominant period of oscillation ranges from 42 to $56 \mathrm{~h}$. The amplitude of oscillation is from $0.1 \mathrm{MHz}$ to $1 \mathrm{MHz}$. The activity of the quasi-2-day oscillation is better expressed during the summer half year when several enhancements, about 15-30 days in duration, were observed. The largest enhancements of the oscillation occurred during early June, July and early August; i. e., near and after the summer solstice when the 2-day wave in the middle neutral atmosphere typically displays its largest activity in the Northern Hemisphere. The results obtained may help us understand better the possible influencing mechanisms between the 2-day wave in the middle neutral atmosphere and the ionospheric quasi-2-day oscillations.
\end{abstract}

Key words. Ionosphere (Ionosphere - atmosphere interactions; Mid-latitude ionosphere; Plasma waves and instabilities)
Correspondence to: D. Altadill

E-mail: ebre.daltadill@readysoft.es Fax: + 34-77-504660

\section{Introduction}

The 2-day wave in the middle neutral atmosphere is presumed to be forced from the lower atmosphere. It has been suggested (Salby, 1981) that the wave is a manifestation of the mixed Rossby-gravity normal mode $(3,0)$. Plumb (1983) proposed that the 2-day wave in the middle neutral atmosphere is due to a baroclinic instability above the summer easterly jet. Wu et al. (1996) studied wave events during January 1993 and July-August 1993 and suggested that the winter planetary waves may be responsible for the summer 2-day wave. Their results suggest also that the 2-day wave is a manifestation of both a global normal Rossby mode wave and a local unstable wave.

The 2-day wave in the mesosphere/lower thermosphere displays maximum activity during summer and its amplitude maximizes about 1 month after solstice in the summer hemisphere (Craig and Eldford, 1981). The wave has a westward prevailing direction of propagation with a zonal wave number $K=3$ (Rodgers and Prata, 1981). It has been shown that the 2-day wave tends to be locked in-phase with local time and linked with a particular region of the globe (Poole, 1990). The annual variation of the dominant period shows maximum in summer and minimum in winter (Harris and Vincent, 1993).

A wide number of investigations have considered these quasi-2-day oscillations in the ionosphere. Mainly these works have studied the existence and temporal behaviour of such oscillations in the $\mathrm{F}$ region electron maximum variations (Pancheva, 1988; Pancheva et al., 1994; Apostolov et al., 1994; and references herein).

The temporal behaviour of the amplitude, period and probability of existence of these quasi-2-day oscillations in the critical frequency $f_{\mathrm{oF}} 2$ at middle latitudes has been found (Apostolov et al., 1995; Altadill et al., 1997). The amplitude of oscillation is modulated by the longterm solar and geomagnetic activity variations, and by the semi-annual and 12-month hemispheric geomagnetic 
waves. The dominant period and the probability of existence display a clear annual variation, with maximum values in summer and minimum in winter. The phase shows a high degree of stability during intervals ranging from 15 days to about 3 months in duration. There are three types of quasi-2-day oscillations in $f_{\mathrm{oF}}$ : (1) westward travelling planetary waves with zonal wave number $K=1$, (2) stationary planetary waves (with unknown wave number), and (3) independent oscillations, with different dominant periods, in locations separated on relatively long distances.

Due to the similarities between the period and activity of the quasi-2-day oscillation in the upper ionosphere and those of 2-day wave in the middle neutral atmosphere, many case studies have been considered a possible coupling of the 2-day oscillations between both atmospheric regimes, mesosphere/lower thermosphere and upper ionosphere. Several potential mechanisms have been proposed. Mikhailov's (1983) model shows that the vertical transport of minor constituents $[\mathrm{O}]$ and $\left[\mathrm{O}_{2}\right]$ generated by 2-day waves can cause dramatic changes in the electron concentration maximum of the F2 layer $(N m \mathrm{~F} 2)$. Pancheva and Lysenko (1988) proposed that the 2-day wave in the meteor wind induces a quasi-2-day oscillation in the electric current system (Ito et al., 1986). The latter can produce such an oscillation in the vertical plasma drift that quasi-2-day oscillations can be generated in the $N m$ F2. A similar mechanism has been proposed by Chen (1992) to explain the 2-day modulation in the equatorial ionization anomaly. Both mechanisms can explain the similar annual variations of the period and probability of existence between the middle atmosphere planetary 2-day wave and the foF2 2-day oscillations. Nevertheless, neither of them can explain entirely why the wave numbers should be $K=3$ in the mesosphere and $K=1$ in the upper ionosphere. Apostolov et al. (1995) proposed a triggering mechanism to explain the different wave numbers observed in both atmospheric regimes: the development of the quasi-2-day oscillation in $f_{\mathrm{OF}} 2$ can be induced by the forcing of such an oscillation in the mesosphere, linked with a particular region of the globe, with further independent development in the $\mathrm{F}$ region. In a case study, Forbes et al. (1997) pointed out that the interaction between the quasi-2-day wave in the mesosphere/lower thermosphere winds and the strong diurnal/semidiurnal dependence of the ionosphere may bring about the different wave numbers.

The purpose of this investigation is to show a new investigatic method for the quasi-2-day oscillations in the ionosphere. The methodology used here allows the study of the existence and development of such oscillations in the ionospheric plasma frequency variations at fixed heights.

\section{Data and analysis}

One-year of hourly ionograms, obtained with a DGS256 ionosonde at Observatori de l'Ebre $\left(40.8^{\circ} \mathrm{N}, 0.5^{\circ} \mathrm{E}\right)$ during 1995, have been used for the study. The time series contains 8522 ionograms, that have been analyzed to obtain true height profiles by applying the ionogram's inversion technique described by Huang and Reinisch (1996). In this technique, the data of each ionospheric layer are fitted with a function of shifted Chebyshev polynomials, so that profiles are obtained up to the F2 layer maximum. The E layer is modeled when traces in the ionogram do not exist (at night time), and a model is adapted for the valley regions. This technique has been validated through comparisons with incoherent scatter radar profiles and comparing the ionograms recalculated from the true height profiles with the original ionograms (Huang and Reinisch, 1996).

Since the starting frequency of the sounder is 1.0 $\mathrm{MHz}$, we did not consider plasma frequencies lower than this value in the true height profiles obtained. The information given by the inversion algorithm below this limit only depends on the model that has been used. This avoids considering the appearance of the E layer at night time. In this way, we obtain the time series of hourly values of plasma frequency, at fixed heights in the selected altitude range, from $170 \mathrm{~km}$ to $220 \mathrm{~km}$ at $10 \mathrm{~km}$ steps. Thus, we are left with six time series. We choose these height limits because there are very few gaps in the continuity of the data. In the lower altitudes beyond the selected range, the quantity of gaps begins to be noticeable during the night due to the fact that there is no E layer; and for altitudes above this range, gaps appear because some-times the F2 maximum is around $220-240 \mathrm{~km}$. By this altitude range limitation, the study is restricted to the main part of the $\mathrm{F}$ region below the F2 peak. The few missing values (because of sounder failure or the limitation cited) are replaced by a linear interpolation between the preceding and following values. After this procedure, each of the six time series contains 8760 hourly values of plasma frequencies recorded at UT.

In this work, the periodogram described by Kuklin (Vitinsky et al., 1986), auto-correlation and complex demodulation (Bloomfield, 1976) analyses have been used for studying the existence and time behaviour of the quasi-2-day oscillations in the ionospheric plasma frequency at fixed heights.

\section{Manifestation of the quasi-2-day oscillations in the plasma frequency at fixed heights}

The preliminary estimations concerning the existence of these quasi-2-day oscillations are made by periodogram analysis with high spectral resolution. The amplitude spectra of time series are found for the whole year and for time intervals corresponding to typical winter, summer and equinoctial months. Figure 1 shows the amplitude spectra of the plasma frequency (at $210 \mathrm{~km}$ altitude) in the periodic range from 20 to $90 \mathrm{~h}$ using a period step of $0.2 \mathrm{~h}$ for: (a) the whole year 1995, (b) January, (c) April, and (d) July 1995. The amplitude spectra of the plasma frequency for the rest of the altitudes are qualitatively the same. 

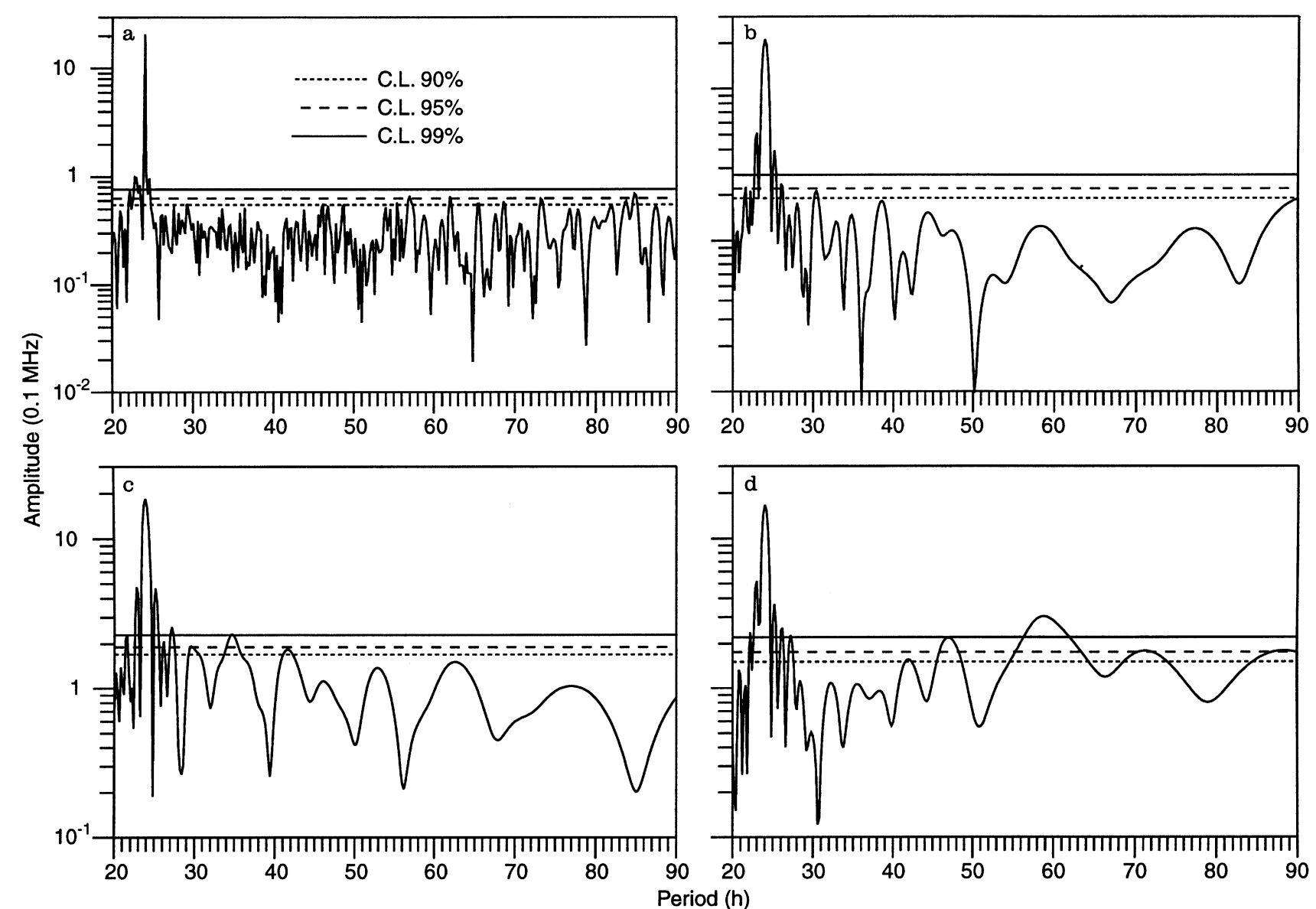

Fig. 1a-d. Amplitude spectra of the plasma frequency variations at $210 \mathrm{~km}$ altitude for: a the whole year 1995, b January, c April, and d July 1995. The horizontal lines are the confidence levels given in the legend

Apostolov et al. (1995) show that the period of the quasi-2-day oscillation in $f_{\mathrm{oF}} 2$ varies between 42 to $56 \mathrm{~h}$. Thus, we can expect a similar period range for the quasi-2-day oscillation in the ionospheric plasma frequency. From Fig. 1a, we observe that the spectral peaks at about $47-49 \mathrm{~h}$ reach the $90 \%$ confidence level (CL) and those about $52-56 \mathrm{~h}$ reach the $95 \% \mathrm{CL}$. This result shows the statistical significance of the quasi-2day oscillation during 1995 . The temporal variations of the amplitude and period of the oscillation can be observed by comparing the different amplitude spectra obtained for winter, equinox and summer. The quasi-2day oscillation is well developed during summer, Fig. 1d shows that the spectral peak at $42 \mathrm{~h}$ is higher than the $90 \% \mathrm{CL}$, that of $48 \mathrm{~h}$ reaches the $99 \% \mathrm{CL}$, and the peak at $58 \mathrm{~h}$ goes well beyond the $99 \% \mathrm{CL}$. During winter, Fig. $1 \mathrm{~b}$ shows that the peaks at 45 and $58 \mathrm{~h}$ are near the $90 \%$ CL. During equinox, (Fig. 1c) the peak at $42 \mathrm{~h}$ reaches $95 \% \mathrm{CL}$ and that at $58 \mathrm{~h}$ is near $90 \% \mathrm{CL}$.

\subsection{Persistence of the quasi-2-day oscillations}

The persistence of the quasi-2-day oscillations in the plasma frequency variations is evaluated by using moving spectral analysis. The time series of plasma frequency are divided into 365 -h intervals shifted by 120-h (5 day) relative to each other. We choose this interval size as an optimum between two requirements: (1) the 2-day oscillations are quasi-periodic and an increase of the interval's size can produce an increase of the ambiguity in the spectral estimates, (2) the size of 365 -h interval is more than 7 times the 48 -h period, which ensures reliable determination of spectral estimates in the periodic range of the quasi-2-day oscillation.

The amplitude spectra have been computed, for each interval and for all time series, in the periodic range from 30 to $80 \mathrm{~h}$ using a period step of $0.2 \mathrm{~h}$. For an accurate determination of the dominant periods, the averaged amplitude spectra and the percentage occurrence of the spectral peaks has been calculated for all altitudes. From Fig. 2, it can be seen that the averaged amplitude spectra (solid line) show spectral peaks at the periods near $31,34,37,42,47-48,53-55$ and $63-65 \mathrm{~h}$ for all altitudes; and the maxima of the percentage occurrence of periods (histograms) take approximately the same values.

The investigation of Altadill (1993) into modulation effects in the time variations of $f_{\mathrm{oF}} 2$ in the periodic range of $30-200 \mathrm{~h}$, leads to the conclusion that some spectral lines with periods from 30 to $65 \mathrm{~h}$ can be the 


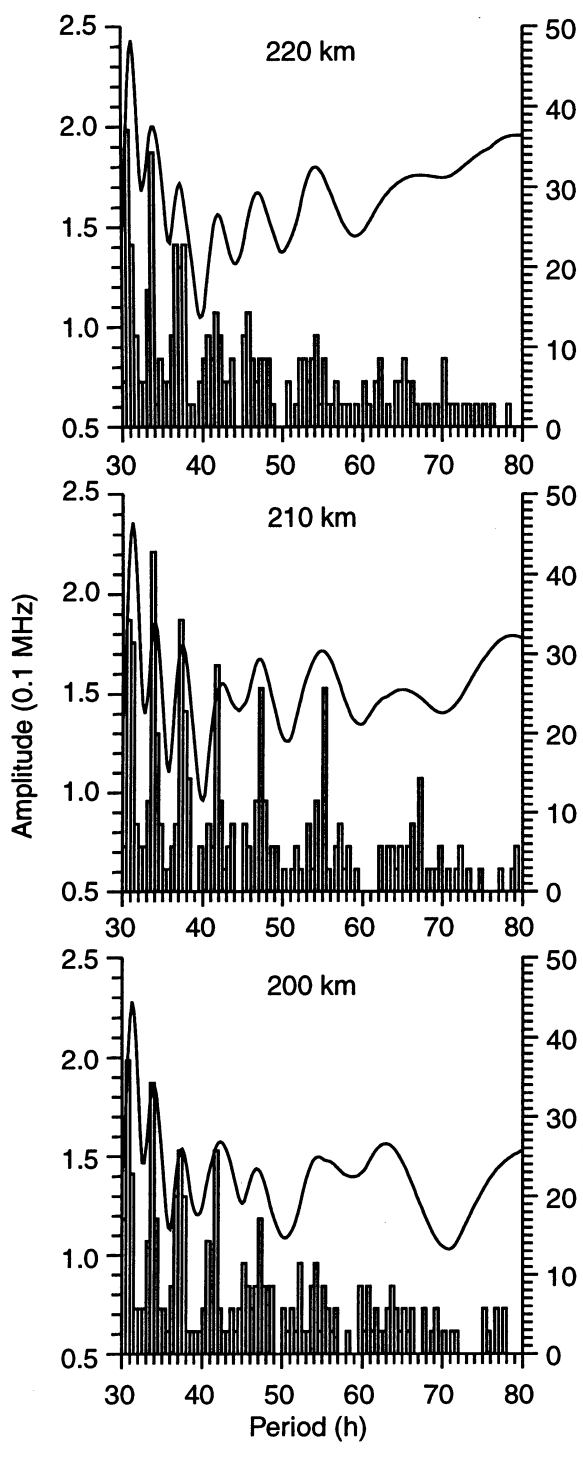

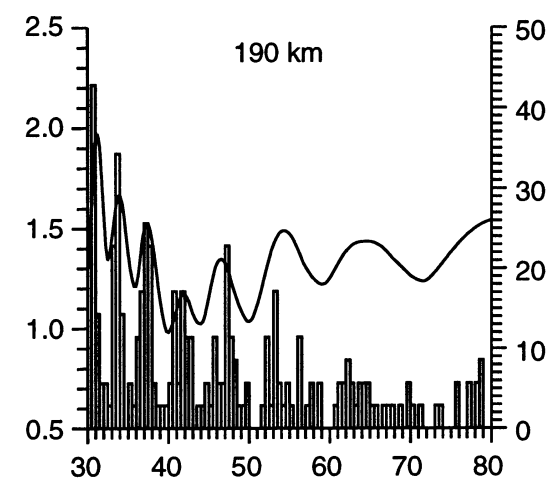
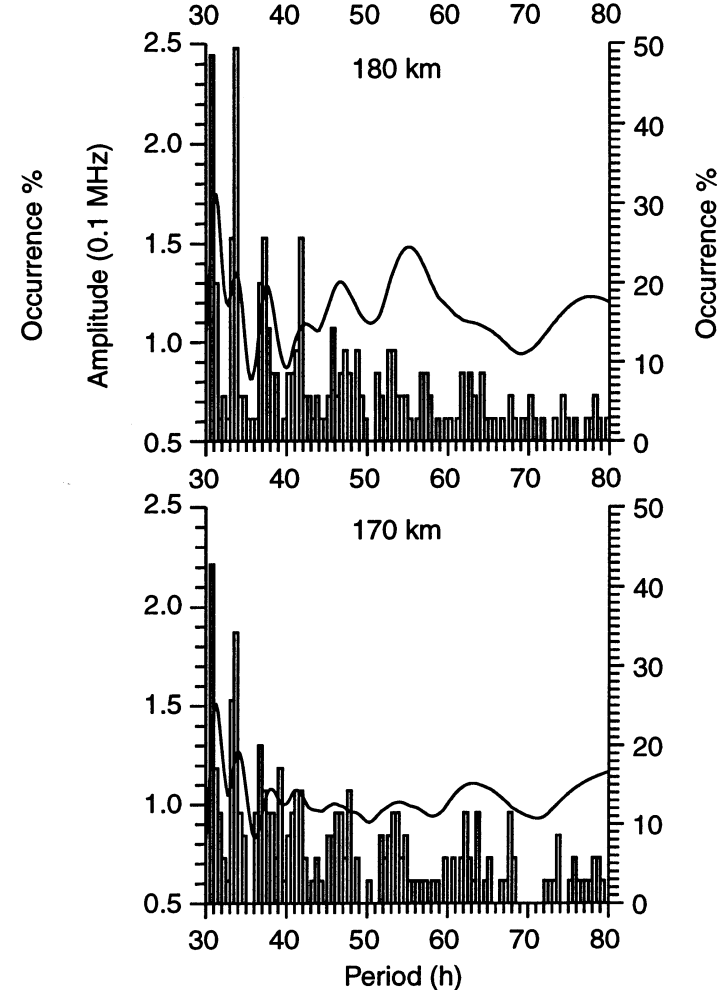

Fig. 2. Averaged amplitude spectra (solid line) and percentage occurrence of periods (histograms) of ionospheric plasma frequency variations during 1995. The left $y$-axis represents the averaged amplitude and the right $y$-axis the percentage occurrence result of amplitude modulation of the quasi-2-day and 3-day oscillations by a modulating period of about 15 days. A quasi-3-day oscillation activity, with a period about $72-78 \mathrm{~h}$, has been detected in the ionospheric plasma frequency variations (Figs. 1, 2). We found also intensive spectral peaks ranging from 13 to 16.5 days (the periodograms for the period range from 200 to $700 \mathrm{~h}$ are not shown here). These spectral peaks with about 15 days period, are possibly related with the solar activity (Donelly and Puga, 1990) and with the 16-day wave into the ionosphere (Parish et al., 1994). Considering the quasi-2-day (47-49 h) and 3-day (72-78 h) as fundamentals, simple calculations show that the other spectral lines of $31,34,37$, and 63-65 h, observed here, may be the result of amplitude modulation by a period of 15-days. The amplitudes of spectral lines resulting from the modulations depend on the modulation depth, and often these amplitudes are larger than those of fundamental periods.

Note that the averaged amplitude of the plasma frequency quasi-2-day oscillations over the whole of 1995 is small, about $0.15 \mathrm{MHz}$ for all altitudes (except at
$170 \mathrm{~km}$, where it reaches only $0.1 \mathrm{MHz}$ ), but greater than the frequency resolution of the sounder. Note also that 1995 is a year of low solar activity, so it is expected that the amplitudes of the 2-day oscillation in the upper ionosphere are small (Apostolov et al., 1994). Considering this we can say that the period of quasi-2-day oscillations in the plasma frequency lies between 40 and $58 \mathrm{~h}$, with dominant values of 42, 47-48 and 53-55 h, which are clearly expressed by spectral peaks in Fig. 2 .

\subsection{Statistical reliability of the plasma frequency quasi-2- day oscillations}

Auto-correlation analysis and periodogram analysis, enabling evaluation of the probability of existence of periods under study, are used in order to study the reliability of the quasi-2-day oscillation in the plasma frequency at fixed heights.

Using the same procedure as in Sect. 3.1, the time series were divided into $365-\mathrm{h}$ intervals shifted by $120-\mathrm{h}$ relative to each other. The periodograms for each 
interval and for all time series are computed in the periodic range from 42 to $56 \mathrm{~h}$ using a period step of $0.1 \mathrm{~h}$ to obtain the probability of existence of spectral peaks. We selected this period range in agreement with the dominant periods obtained by the averaged amplitude spectra and percentage occurrence (Sect. 3.1), and according to the expected quasi-2-day period deduced from previous works cited in the text. The percentage intervals (corresponding to each time series) for which the probability of existence, $P$, of periods in the selected range is located between the given limits, are presented in Table 1. This shows that oscillations with a period between 42 and $56 \mathrm{~h}$ exist in the plasma frequency at fixed heights for about $40 \%$ of the time intervals with probability $P>0.8$ and for about $20-30 \%$ of the time intervals when $P>0.9$. From these results, we have evaluated the annual variation of $P$ of the 2-day oscillation in the ionospheric plasma frequency as an average of the annual variations of the probability of existence over the six altitude series. This result is plotted in Fig. 3. This clearly shows that the 2-day oscillation in the ionospheric plasma frequency exists with greatest probability during summer, from June to

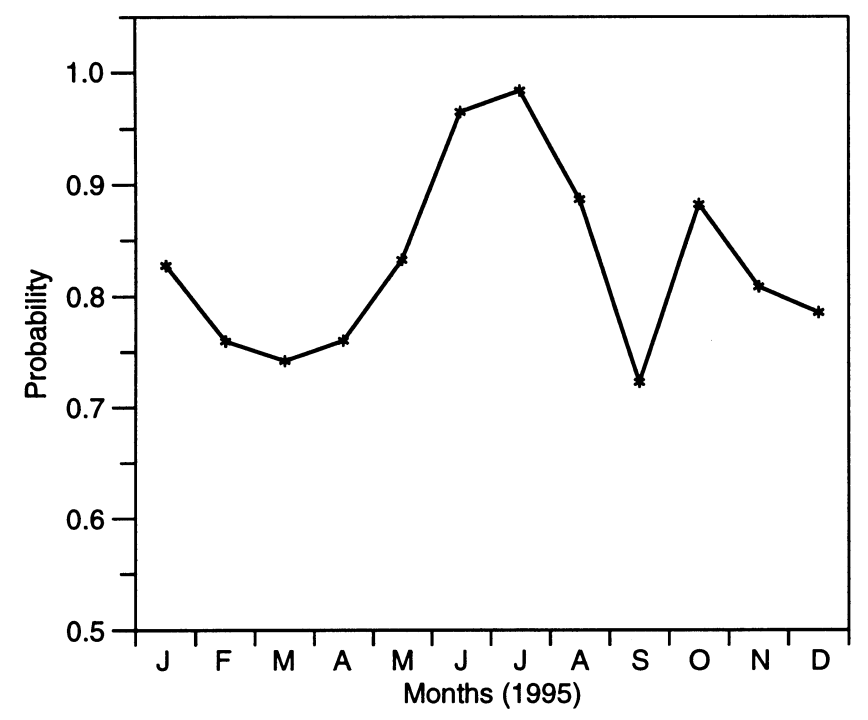

Fig. 3. Annual variation of the existing probability of the 2-day oscillation in the ionospheric plasma frequency
August. A significant enhancement during October is also observed.

The auto-correlation function shows us whether periodicity is real or not. Previous to the auto-correlation analysis, the quasi-2-day oscillation had been isolated with a band-pass filter centred at $48 \mathrm{~h}$ with bandwidth of $13 \mathrm{~h}$. Figure 4 (left panel) shows the filtered data obtained from May 24 to August 22 of 1995 (i. e. 90-day interval) for the studied altitude range. Note that in every plot of Fig. 4 (left) there are from 44 to 46 oscillations, that correspond to a period near 2 days. Figure 4 (left) shows that the amplitude modulation of the quasi-2-day oscillations is similar for the different altitudes, which is clearly seen in the several intervals of enhanced 2-day oscillations. Small differences can be observed in the amplitude modulation of the quasi-2day oscillation for altitudes of 170 and $180 \mathrm{~km}$ respect to the others. Note that at these altitudes the transition region between F1 and F2 layers is often located. Note also that very often the amplitude is larger than $0.1 \mathrm{MHz}$ and, during the intervals of enhanced oscillation, the amplitude reaches values near $1 \mathrm{MHz}$.

The auto-correlation functions of the filtered time series and the 95\% CL were computed and plotted in Fig. 4 (right panel) for the different altitudes and for the summer time interval cited already. The auto-correlation functions were obtained from 0 up to $720 \mathrm{~h} \mathrm{lag.}$ From Fig. 4 (right), we observe that the quasi-2-day oscillation of the plasma frequency persists with probability higher than $95 \%$ and the peaks of auto-correlation functions repeat every time lag of $48 \mathrm{~h}$. The results of auto-correlation analysis show that the quasi-2-day oscillations are real and statistically significant in the plasma frequency variations for all studied altitudes during summer.

\section{Time behaviour of oscillation activity during 1995}

To estimate in detail the temporal amplitude variations of the 2-day oscillation in the plasma frequency, we use the complex demodulation of time series method. Previous to the complex demodulation analysis, the 2day oscillations in the plasma frequency time series are isolated by the band-pass filter with the same charac-

Table 1. Percentage of 365-h intervals over an altitude range from 170 to $220 \mathrm{~km}$, for which the probability $P$ of existence of spectral peaks in the periodic range from 42 to $56 \mathrm{~h}$ is located between the given limits of probability

\begin{tabular}{|c|c|c|c|c|c|c|}
\hline & \multicolumn{6}{|c|}{ Altitude (km) } \\
\hline & 170 & 180 & 190 & 200 & 210 & 220 \\
\hline $0.50<P \leq 0.55$ & 5.7 & 1.4 & 4.3 & 1.4 & 7.1 & 5.7 \\
\hline $0.55<P \leq 0.60$ & 2.9 & 2.9 & 4.3 & 10.0 & 4.3 & 4.3 \\
\hline $0.60<P<0.65$ & 11.4 & 5.7 & 5.7 & 8.6 & 4.3 & 4.3 \\
\hline $0.65<P \leq 0.70$ & 8.6 & 2.9 & 7.1 & 8.6 & 2.9 & 7.1 \\
\hline $0.70<P \leq 0.75$ & .0 & 11.4 & 5.7 & 4.3 & 10.0 & 5.7 \\
\hline $0.75<P \leq 0.80$ & 5.7 & 7.1 & 11.4 & 2.9 & 12.9 & 5.7 \\
\hline $0.80<P \leq 0.85$ & 2.9 & 8.6 & 5.7 & 4.3 & 8.6 & 11.4 \\
\hline $0.85<P \leq 0.90$ & 14.3 & 2.8 & 5.7 & 10.0 & 2.9 & 14.3 \\
\hline $0.90<P \leq 0.95$ & 11.4 & 8.6 & 8.3 & 4.3 & 5.7 & 2.9 \\
\hline $0.95<P \leq 1.00$ & 20.0 & 25.7 & 15.7 & 15.7 & 22.9 & 18.6 \\
\hline
\end{tabular}




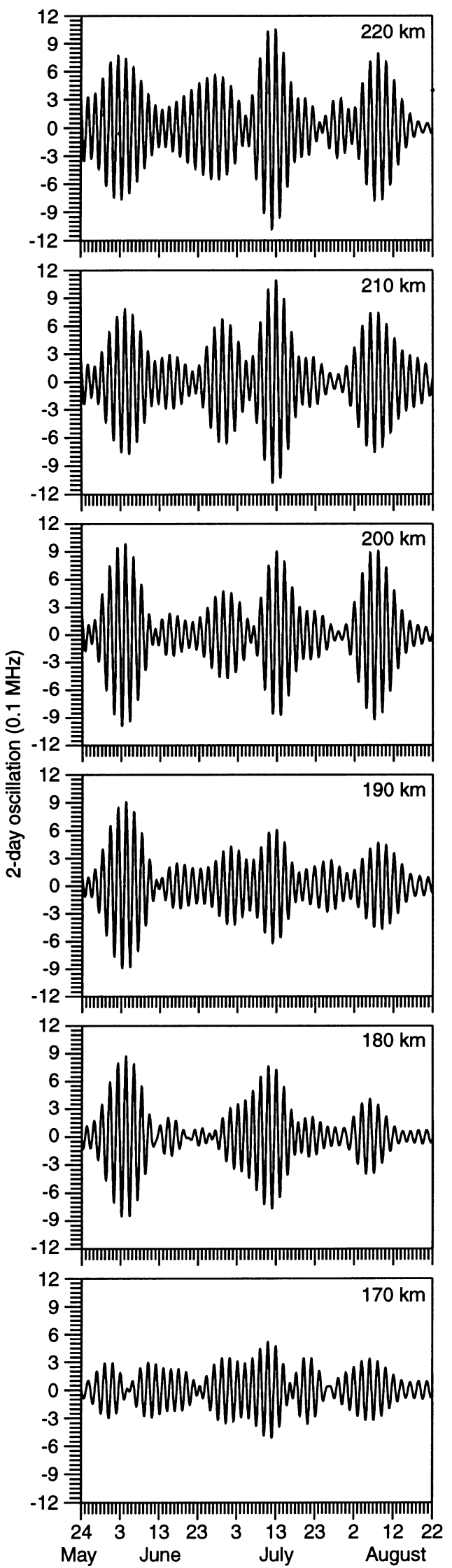

Fig. 4. The left panel shows the filtered data of the plasma frequency at fixed heights corresponding to the quasi-2-day oscillation obtained from May 24 to August 22 of 1995. The right panel displays the auto-
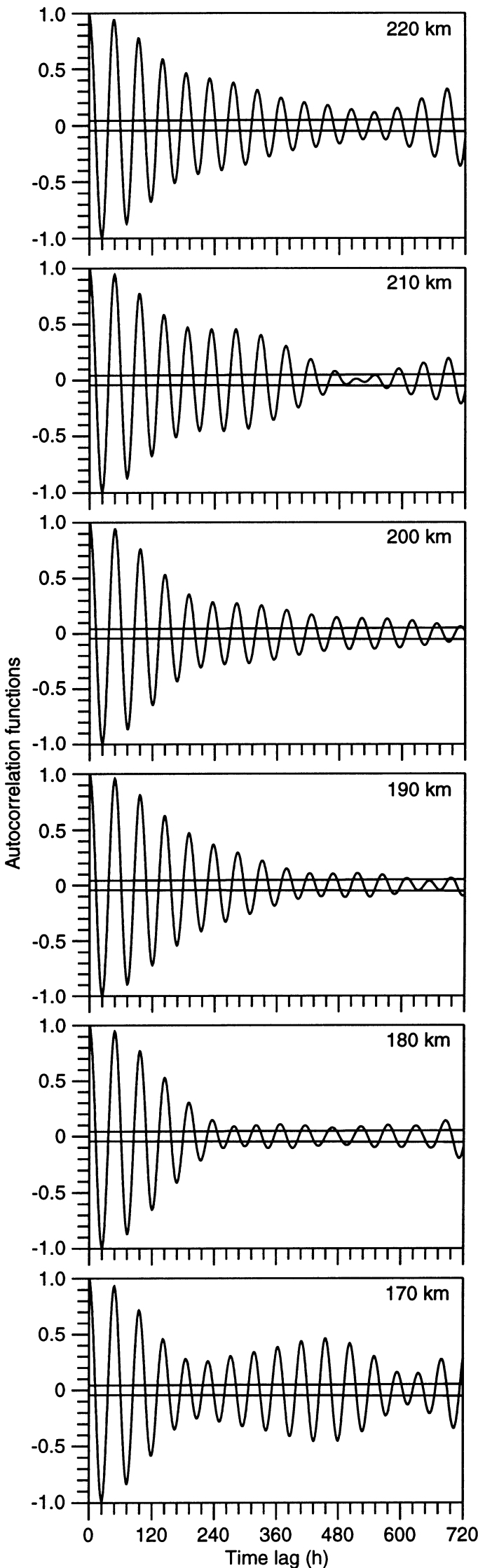

correlation functions of the filtered data (heavy lines) and their corresponding 95\% confidence levels (thin lines) for the same time interval 
teristics as noted already. A demodulating period $T_{d}=48 \mathrm{~h}$ for complex demodulation of all time series was applied. In the procedure of complex demodulation, a low-pass filter with cutoff period $T_{c}=72 \mathrm{~h}$ and 143 least squared terms is used to remove the perturbed sinusoid of the second harmonic of $T_{d}$ (Bloomfield, 1976). The instantaneous amplitudes obtained by complex demodulation, are plotted in Fig. 5 (solid line) for the altitudes indicated. From Fig. 5 it can be seen that amplitudes of quasi-2-day oscillations in the ionospheric plasma frequency at fixed heights range from 0.1 to $1 \mathrm{MHz}$. Several intervals of enhancement of the quasi-2day oscillations, with a duration of about 15 to 30 days, associated with a significant increase of amplitude can be observed during April, May-June, July, August and October for all heights investigated. The largest enhancements of the oscillation occur during early June, July and early August; i. e., near and after the summer solstice when the 2-day wave in the middle neutral atmosphere typically displays larger activity in the Northern Hemisphere. The enhancements of the quasi2-day oscillations, with an increase of the amplitude during 10-30 days (i.e. 5-15 oscillations) in separate time intervals, are a typical feature of the annual variation of the quasi-2-day wave activity in the mesosphere/lower thermosphere winds also (Harris and Vincent, 1993).

The relative energy contributions of the quasi-2-day oscillation to the energy of the plasma frequency variations for the periods from 2 to $58 \mathrm{~h}$ (i. e., including the daily variation) have been obtained. The relative energy contributions have been evaluated using the methodology described by Parish et al. (1994), as the percentage ratio of the spectral energy of the 2-day oscillation (the periodic band from 40 to $58 \mathrm{~h}$ ) to the spectral energy of the periodic variations from 2 to $58 \mathrm{~h}$.

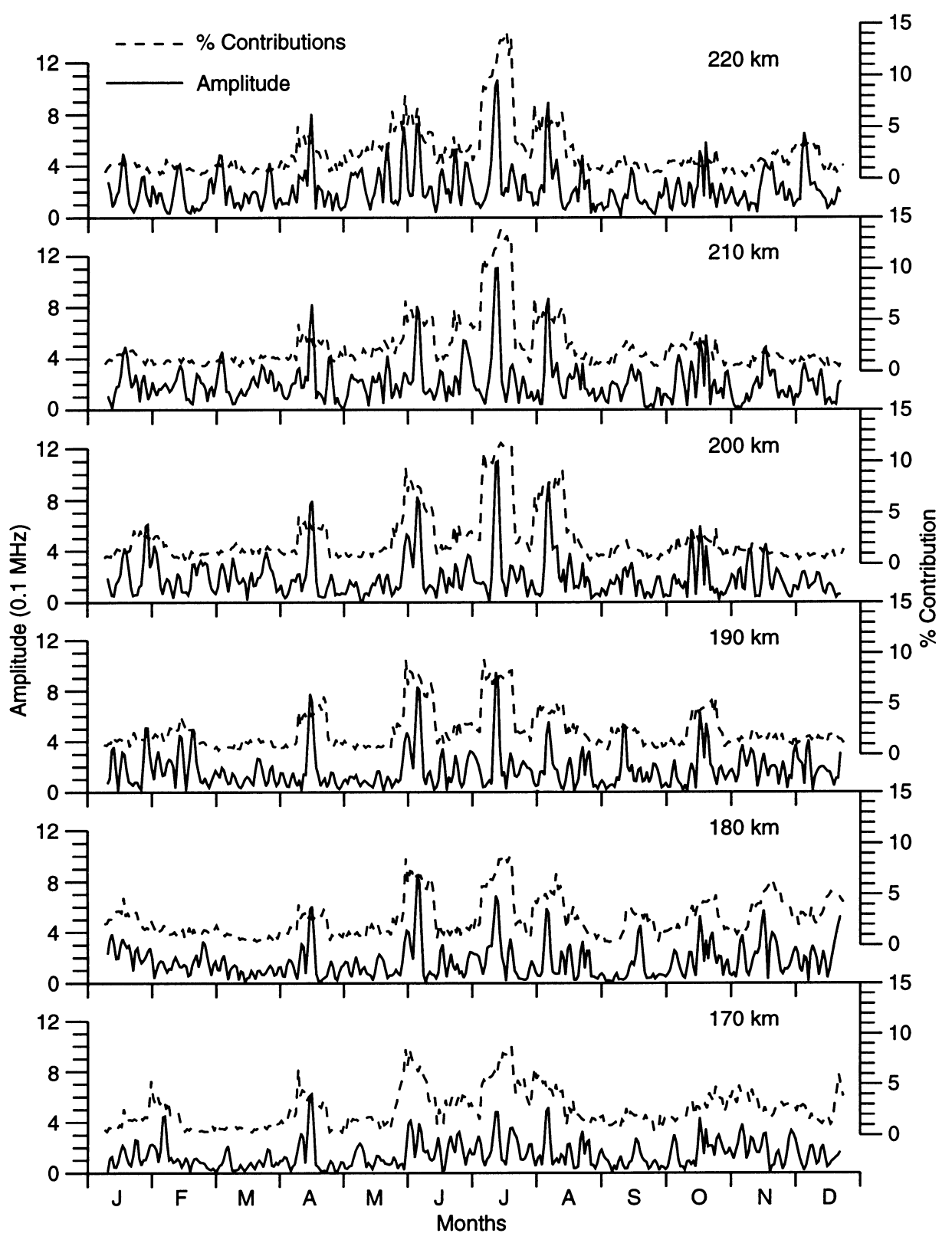

Fig. 5. Annual variations of the instantaneous amplitude (solid line) and percentage contributions of spectral energy (dashed line) for the quasi-2-day oscillation in the ionospheric plasma frequency during 1995. The left y-axis represents the quasi-2-day amplitude and the right $y$-axis their spectral energy contribution 


$$
\text { Contribution }(\%)=\frac{\int_{40}^{58}|X(T)|^{2} \mathrm{~d} T}{\int_{2}^{58}|X(T)|^{2} \mathrm{~d} T} * 100
$$

where $|X(T)|^{2}$ refers to the energy density spectrum, which is obtained here by periodogram analysis.

The contributions are calculated by periodograms in the periodic range from 2 to $58 \mathrm{~h}$ for successive time intervals of 365 - $\mathrm{h}$ shifted by $24 \mathrm{~h}$ to each other. The annual variations of the percentage contribution of the quasi-2-day oscillation for each fixed height are presented in Fig. 5 (dashed line). The percentage contribution of quasi-2-day oscillation for the largest pulse observed in July increases with height from $8 \%$ at $170 \mathrm{~km}$ to $14 \%$ at $220 \mathrm{~km}$. The percentage contributions for the other enhancements of the oscillation are about 3-7\% and they remain nearly equal at all altitudes. The annual variation of the relative energy contributions of the quasi-2-day oscillation, obtained here, can be interpreted as the annual variation of the quasi-2-day oscillation activity with respect to the activity of the total daily variation in the ionospheric plasma frequency. Taking into account the fact that the amplitude and the spectral energy of the daily variations are predominant in the ionospheric plasma frequency variations, the percentage contribution of the quasi-2-day oscillations to the total energy from $3-7 \%$ to $14 \%$ shows a significant contribution of this oscillation to the variability of electron density at fixed heights. From these results, the largest enhancements of the quasi-2-day oscillation are clearly expressed in the annual variations of both, the amplitude and the spectral energy contribution. It is also observed that the largest activity of the quasi-2-day oscillation in the ionospheric plasma frequency have a tendency to occur during the summer half year, at which time the maximum activity of the 2-day wave in the middle neutral atmosphere is expected.

\section{Concluding remarks}

The quasi-2-day oscillation has been observed in the ionospheric plasma frequency (or electron density) variations at fixed heights. These oscillations persist almost continuously during the whole of 1995 and they are statistically significant during summer. The oscillation characteristics behaviour are in agreement with the characteristics of the quasi-2-day oscillation observed in the $f_{\mathrm{oF}} 2$. The largest activity of the quasi-2-day oscillation in the ionospheric plasma frequency during 1995 occurs in the summer half year. Enlarged amplitudes of the quasi-2-day oscillation, associated with several pulses of 10-30 days duration, have also been observed. During the intervals of enhancement, the contribution of the quasi-2-day oscillation to the variability of electron density is very significant. The intervals of largest enhancement of the quasi-2-day oscillation in the plasma frequency occur near and after the summer solstice when the 2-day wave in the middle neutral atmosphere typically displays larger activity in the Northern Hemisphere. These enhancements of the quasi-2-day oscillations is a typical feature of the annual variation of the 2-day wave activity in the mesosphere/ lower thermosphere winds also. Thus, the temporal behaviour of the quasi-2-day oscillation activity in the ionospheric plasma frequency observed here as a "burst", which is similar to that of the middle neutral atmosphere 2-day wave, suggests a possible coupling of both atmospheric regimes by the 2-day oscillations.

The methodology described enables the study of the altitude development of the quasi-2-day oscillations in the ionosphere. The results obtained by this methodology can give more information about the quasi-2-day oscillation in the ionosphere than the results obtained in previous investigations of $f \mathrm{oF} 2$. Consequently, this methodology can help to understand better the start and development of the quasi-2-day oscillation in the ionosphere compared to the 2-day wave in the middle neutral atmosphere, as well as to evaluate the priorities of the currently proposed mechanisms. Also, considering these potential mechanisms and assuming a vertical propagation of the quasi-2-day oscillations into the ionosphere, the altitude development of the spectral energy contribution can suggest the energy deposition of the oscillation into the ionosphere.

The results presented here are not able to throw a light onto the mechanism responsible for the 2-day oscillations in the ionosphere. For this purpose, simultaneous studies of 2-day oscillation in the middle neutral atmosphere and in the ionosphere at fixed heights are needed. This will be the aim of our next investigation.

Acknowledgements. Authors wish to thank Dr. Bodo W. Reinisch for providing ionograms' inversion software packages. E. M. Apostolov wishes to express his appreciation to the Program Cathedra of Bank Bilbao Vizcaya Foundation for supporting him during three months stay in 1997 at Observatori de l'Ebre.

Topical Editor D. Alcaydé thanks J. Dudeney and A. H. Manson for their help in evaluating this paper.

\section{References}

Altadill, D., Possible amplitude modulation effects in the $f_{\mathrm{oF}} 2$ variations in the periodic range 30-200 hours, Bulg. Geophys. J., 19, 26-31, 1993

Altadill, D., E. M. Apostolov, and L. Alberca, Some seasonal hemispheric similarities in $f_{\mathrm{oF}} 2$ quasi-2-day oscillations, $J$. Geophys. Res., 102, 9737-9739, 1997.

Apostolov, E. M., L. Alberca, and D. Altadill, Solar cycle and seasonal behaviour of quasi-two- and five-day oscillations in the time variations of foF2, Ann. Geofis, 37, 187-192, 1994.

Apostolov, E. M., D. Altadill, and L. Alberca, Characteristics of quasi-2-day oscillation in $f_{\mathrm{OF}} 2$ at northern middle latitudes, $J$. Geophys. Res., 100, 12162-12171, 1995.

Bloomfield, P., Fourier analysis of time series: an introduction, John Wiley, New York, 1976.

Craig, R. L., and W. G. Eldford, Observation of the quasi two-day wave, J. Atmos. Terr. Phys., 43, 1051-1056, 1981.

Chen, P.-R., Two-day oscillation in the equatorial ionization anomaly, J. Geophys. Res., 97, 6343-6357, 1992.

Donelly, R. F., and L. C. Puga, Thirteen-day periodicity and the centre-to-limb dependence of UV, EUV and X-ray emission of solar activity, Sol. Phys., 130, 369-390, 1990. 
Forbes, J. M., R. Guffe, X. Zang, D. Fritts, D. Riggin, A. Manson, C. Meek, and R. A. Vincent, Quasi 2-day oscillation of the ionosphere during summer 1992, J. Geophys. Res., 102, 7301$7305,1997$.

Harris, T. J., and R. A. Vincent, The quasi-two-day wave observed in the equatorial middle atmosphere, J. Geophys. Res., 98, 10481-10490, 1993.

Huang, X., and B. W. Reinisch, Vertical electron density profiles from the digisonde network, Adv. Space Res., 18, 121-129, 1996.

Ito, R., S. Kato, and T. Tsuda, Consideration of an ionospheric wind dynamo driven by a planetary wave with a two-day period, J. Atmos. Terr. Phys., 48, 1-13, 1986.

Mikhailov, A. V., Mechanism of in phase variations of electron concentration between E and F2 ionospheric layers (in Russian), Geomag. Aeron., 23, 557-561, 1983.

Pancheva, D., Travelling quasi-two-day fluctuations in the summer F-region, C. R. Acad. Bulg. Sci., 41, 41-44, 1988.

Pancheva, D., and I. Lysenko, Quasi two-day fluctuations observed in the summer F region electron maximum, Bulg. Geophys., J., 14, 41-51, 1988.

Pancheva, D., L. Alberca, and B. de la Morena, Simultaneous observations of quasi-two-day variations in the lower and upper ionosphere over Europe, J. Atmos. Terr. Phys., 56, 43-50, 1994.
Parish, H. F., J. M. Forbes, and F. Kamalabadi, Planetary wave and solar emission signature in the equatorial electrojet, J. Geophys. Res., 99, 355-368, 1994.

Plumb, A. R., Baroclinic instability of the summer mesosphere: a mechanism for the quasi-two-day wave?, J. Atmos. Sci., 40, 262-270, 1983.

Poole, L. M. G., The characteristics of the mesospheric two-day wave as observed at Grahamstown $\left(33.3^{\circ} \mathrm{S}, 26.5^{\circ} \mathrm{E}\right), \mathrm{J}$. Atmos. Terr. Phys., 52, 258-269, 1990.

Rodgers, C. D., and A. J. Prata, Evidence for travelling two-day wave in the middle atmosphere, J. Geophys. Res., 86, 96619664, 1981.

Salby, M. L., The 2-day wave in the middle atmosphere: Observations and theory, J. Geophys. Res., 86, 9654-9660, 1981.

Vitinsky, Y. I., M. Kopecki, and G. Kuklin, Statistics of sunspotcreating activity (in Russian), Nauka, Moskow, 1986.

Wu, D. L., E. F. Fishbein, W. G. Read, and J. W. Waters, Excitation and evolution of the quasi-2-day wave observed in UARS/MLS temperature measurements, J. Atmos. Sci., 53, 728-738, 1996. 